\title{
Neutrophil to Leukocyte Ratio
}

National Cancer Institute

\section{Source}

National Cancer Institute. Neutrophil to Leukocyte Ratio. NCI Thesaurus. Code C64827.

The determination of the ratio of neutrophils compared to leukocytes present in a sample. The measurement may be expressed as a ratio or percentage. 